\title{
Influence du rythme de distribution sur les activités alimentaires et l'évolution journalière du contenu ruminal chez le mouton
}

\author{
J. P. DULPHY, Y. ELMEDDAH, R. BAUMONT
}

avec la collaboration technique de Marie JAILLER, L. L'HOTELIER et H. BOUSQUET

Unité Ingestion, Station de Recherche sur la Nutrition des Herbivores, I.N.R.A. Theix, 63122 Ceyrat, France.

Summary. Ingestive activities and diurnal rumen content variations in sheep as affected by feeding frequency.

Three feeding frequencies of late-cut grass hay (two times per day at $8 \mathrm{~h}$ interval, once a day, two times per day at $12 \mathrm{~h}$ interval) were compared in sheep fitted or not with a rumen fistula. The following parameters were recorded: daily dry matter intake, ingestive activities, reticulorumen load before and after the large meal following each feeding.

The daily dry matter intake was not significantly affected by the treatments although it tended to be lower with one only feeding per day.

The animals consumed a larger meal after a single feeding in the morning than after two feedings and they thereafter had more small meals during the day. The total time spent eating and ruminating was similar for the three treatments, always with more time spent ruminating in the night than in the day.

Whatever the feeding interval ( 8 or $12 \mathrm{~h}$ ), the weight of the reticulorumen content was higher after the evening meal.

Le ruminant, au pâturage, effectue deux grands repas par jour, l'un après l'aube et l'autre avant le coucher du soleil (Dulphy, Remond et Theriez, 1979). Entre ces deux grands repas existent des périodes d'ingestion intermédiaires de plus courte durée. En stabulation les animaux alimentés en libre-service effectuent le plus grand nombre de repas en période diurne, avec deux pics, l'un en début, l'autre en fin de journée (Chase, Wangness et Baumgardt, 1976). En revanche, la distribution de l'aliment incite l'animal à manger, même s'il n'a pas ingéré tout ce qui lui avait été distribué précédemment. Le rythme de distribution de l'aliment influence donc l'organisation des activités alimentaires des animaux (Ulyatt et al., 1984 ; Nocek et Braund, 1985).

Dans le cas d'une distribution unique d'aliment le matin, Metz (1975) observe que des vaches effectuent quand même un second repas avant la période d'obscurité. Les animaux étudiés par Nocek et Braund (1985) présentent aussi un pic d'ingestion en milieu d'après-midi. 
L'état de réplétion du réticulo-rumen est lié aux activités d'ingestion. Chez des moutons alimentés 2 fois par jour, à 8 heures d'intervalle, cet état de réplétion est plus important après le repas du soir qu'après celui du matin (Dulphy, Béchet et Thomson, 1975 ; Baumont, Dulphy et Andrieu, 1988). Thomson et al. (1985) observent d'ailleurs la même chose au pâturage. Or, pour des études quantitatives sur la digestion et l'ingestion, il faut connaître le volume du contenu du réticulo-rumen tout au long du nycthémère, ce qui n'est pas aisé avec un rythme de distribution discontinu, comprenant 2 distributions par jour espacées de $8 \mathrm{~h}$, comme nous le faisons habituellement. Le volume du contenu du réticulo-rumen est en effet mesuré après vidages des animaux, vidages qui peuvent les perturber et qui modifient pour quelques heures la stratification du contenu.

Nous avons donc cherché à modifier le rythme de distribution que nous utilisons en faisant deux hypothèses, celle du maintien du niveau d'ingestion de l'aliment et celle de la possibilité de réduire le nombre de vidages nécessaires pour suivre l'évolution du contenu réticulo-omasal.

Pour cela nous avons tout d'abord réduit le nombre de distributions d'aliment à une seule par jour, ce qui correspond au système le plus simple. Nous avons ensuite porté l'intervalle entre deux distributions à $12 \mathrm{~h}$ avec comme idée la réduction de la période d'observation des animaux à $12 \mathrm{~h}$ seulement.

Dans les 3 conditions d'alimentation étudiées nos objectifs étaient donc la mesure simultanée des quantités ingérées, des activités alimentaires et de l'évolution du contenu réticulo-omasal, les observations faites devant, en définitive, nous éclairer sur le rôle de l'état de réplétion du rumen dans le contrôle de l'ingestion journalière (Baumont, Dulphy et Andrieu, 1988).

\section{Matériel et méthodes}

Animaux et aliments. - Huit moutons castrés de race Texel, âgés de 4 à 5 ans, ont été utilisés, dont 4 munis d'une fistule du rumen de $75 \mathrm{~mm}$ de diamètre. Ces animaux pesaient, en moyenne, $70 \mathrm{~kg}$. Ils ont reçu un foin de dactyle tardif, séché au sol dans de bonnes conditions, et dont les caractéristiques étaient les suivantes, en $\mathrm{g} / \mathrm{kg}$ de $\mathrm{MS}$ :

- matières azotées totales

- cendres

- cellulose brute de Weende

- NDF de Van Soest

- ADF de Van Soest

Schéma expérimental. — Durant tout l'essai les animaux ont été nourris à volonté (10\% de refus environ) et ont eu un accès continu au foin, les refus étant toujours enlevés chaque matin à $8 \mathrm{~h}$. Quatre périodes se sont succédées dans le temps: $\mathrm{P} 1$ : 2 distributions journalières à $9 \mathrm{~h}$ et $17 \mathrm{~h} ; \mathrm{P} 2: 1$ distribution à $9 \mathrm{~h} ; \mathrm{P} 3$ : 2 distributions journalières à $9 \mathrm{~h}$ et $21 \mathrm{~h} ; \mathrm{P} 4$ : identique à $\mathrm{P} 1$. 
Chaque période a consisté en une adaptation de 10 jours au rythme de distribution, puis en 2 semaines ( $P 1$ et $P 3$ ), ou une semaine (P2) pour la mesure de l'état de réplétion du rumen, ensuite une semaine pour l'enregistrement des activités alimentaires ( $\mathrm{P} 2, \mathrm{P} 3$ et $\mathrm{P} 4)$ et une autre en $\mathrm{P} 3$ pour la mesure de la digestibilité du foin.

L'essai a été réalisé en période de jours croissants. Pour éviter toute influence de la durée d'éclairement sur les quantités ingérées (Michalet-Doreau et Gatel, 1984), les animaux ont été maintenus éclairés de 6 à $22 \mathrm{~h}$ tous les jours afin de simuler les conditions d'éclairement en jours longs.

Mesures. - Les mesures concernant l'état de rép/étion du réticulo-rumen ont été faites par vidage manuel complet des 4 moutons fistulisés, les animaux étant au sol, dans les conditions décrites par Baumont, Dulphy et Andrieu (1988). Ces vidages, espacés au minimum de 3 jours l'un de l'autre, ont été faits aux heures suivantes: P1 : 9, 11, 17 et $19 \mathrm{~h} ; \mathrm{P} 2: 9$ et $11 \mathrm{~h} 30 ; \mathrm{P} 3: 9,11,21$ et $23 \mathrm{~h}$.

Les activités alimentaires et méryciques ont été enregistrées également selon la technique de Baumont et al. (1988) sur 3 moutons fistulisés et 3 moutons normaux placés en cage à métabolisme. Les mesures ont duré 5 jours après une adaptation de 3 jours aux cages et au matériel d'enregistrement, que les animaux connaissaient déjà.

Les séquences d'activité durant lesquelles les mouvements de mâchoire ont occupé moins de $50 \%$ du temps ont été négligées. Elles représentent, en temps réel d'activité, $33 \mathrm{~min} /$ jour en moyenne. Ce sont des séquences pendant lesquelles les animaux boivent, bêlent, lèchent ou mordillent leur auge. L'ingestion de fourrage pendant ces séquences est donc négligeable.

Les quantités ingérées par jour et lors des grands repas du matin ont été obtenues par différence entre les quantités offertes et refusées. Au niveau de la journée elles ont été mesurées pendant toute la durée de l'essai. Lors des grands repas elles ont été mesurées le jour de chaque vidage, lorsque celui-ci était placé après, et tous les matins lors de l'enregistrement des activités alimentaires.

La digestibilité de la matière organique a été mesurée par collecte totale des fèces pendant 6 jours consécutifs sur les 6 animaux placés en cage à métabolisme, en fin de période P3. Elle a été égale à 0,58 ( $\pm 0,017$ ).

Analyses de laboratoire. - En dehors des jours d'adaptation les teneurs en matière sèche du fourrage offert et refusé, ainsi que celles des fèces en P3, ont été mesurées dans une étuve à $80^{\circ} \mathrm{C}$. Sur un échantillon représentatif du fourrage offert lors de toutes les périodes de mesures les teneurs en cendres (incinération à $550^{\circ} \mathrm{C}$ ), en MAT (méthode Kjeldahl), en cellulose brute de Weende et en parois végétales (Goering et Van Soest, 1970) ont été déterminées.

Sur des échantillons représentatifs des contenus de rumen nous avons mesuré les teneurs en matière sèche, ainsi que celles en parois totales (NDF de Goering et Van Soest), ce pour chaque vidage ( 3 traitements $\times 4$ moutons $\times 2$ ou 4 vidages par traitement pour un mouton). 
Analyses statistiques. - Les effets mouton et rythme de distribution ont été testés par analyse de variance. Nous n'avons retenu ensuite que l'effet du rythme de distribution et les écarts-types résiduels (tabl. 1, 2 et 3).

\section{Résultats.}

\section{Quantités ingérées.}

Les quantités de matière sèche ingérées par jour n'ont pas été significativement affectées par le traitement. Pour les 8 animaux elles ont été égales à $1320 \pm 184,1233 \pm 129,1346 \pm 164$ et $1320 \pm 150 \mathrm{~g} /$ jour, soit $56,50,56$ et $53 \mathrm{~g} / \mathrm{kg} \mathrm{P}^{0,75} /$ jour durant la totalité des périodes P1, P2, P3 et P4 (écart-type résiduel final de $92 \mathrm{~g} / \mathrm{jour}$ et de $3,7 \mathrm{~g} / \mathrm{kg} \mathrm{P}^{0,75}$ ). L'équivalence entre les périodes $\mathrm{P} 1$ et $\mathrm{P} 4$ indique que la capacité d'ingestion des moutons s'est maintenue pendant tout l'essai et que les périodes $\mathrm{P} 2$ et $\mathrm{P} 3$ peuvent être comparées aux autres. Malgré tout l'ingestion est plus faible de $6 \%$ en P2 (une distribution) par rapport à $P 1$ ( 2 distributions). Les quantités journalières ingérées par les moutons fistulés (tabl. 3) ont été identiques à celles des moutons non fistulés, ce qui indique que, ni la fistule, ni les vidages, n'ont perturbé l'ingestion de ces animaux.

\section{Activités alimentaires et méryciques (tabl. 1).}

Les quantités ingérées, lors des journées durant lesquelles étaient enregistrés les mouvements de mâchoire des moutons, ont été pratiquement identiques à celles obtenues pour l'ensemble des jours de mesure de l'ingestion (cf. plus haut). Les différents rythmes de distribution n'ont pas eu d'influence significative sur les durées journalières d'ingestion (en moyenne $345 \mathrm{~min}$ ) et de rumination (en moyenne $526 \mathrm{~min}$ ). Les moutons ont ingéré leur foin plus rapidement en P3. II y a eu plus de petits repas en $\mathrm{P} 2$. Durant cette période l'ingestion a été très groupée le matin et l'après-midi jusqu'à $17 \mathrm{~h}$, mais elle a duré encore 92 min au-delà de $17 \mathrm{~h}$ (tabl. 2). Nous n'avons pas observé de grands repas spontanés l'après-midi, tout au plus un "petit repas" atteignant $60 \mathrm{~min}$ en moyenne et situé à des moments très variables dans la journée.

Les quantités ingérées lors du grand repas du matin ont été différentes selon les traitements : $+31 \%$ en $\mathrm{P} 2$ (une distribution) par rapport à $\mathrm{P} 4$, mais aussi $+12 \%$ en P3 par rapport à P4. Les moutons fistulés ont cependant eu un comportement différent des non fistulés. En effet ils ont ingéré moins (tabl. 3) lors des grands repas précédant les vidages de la fin de matinée et du soir, pendant moins de temps, ce qui a permis de faire les vidages de la fin de matinée en P2 à $11 \mathrm{~h} 30$. Notons aussi que les vitesses d'ingestion lors des grands repas du matin ont été proches d'un traitement à l'autre, en moyenne égales à 4,93 g de $\mathrm{MS} / \mathrm{min}$, malgré une augmentation de $14 \%$ en P3 par rapport à P2.

Les différents traitements ont peu modifié la répartition des activités méryciques (tabl. 2). Le nombre journalier de bols de rumination n'a pas varié non plus d'un traitement à l'autre ( 574 bols en moyenne), ni d'ailleurs la quantité de matière sèche mastiquée par minute d'ingestion et de rumination $(1,52 \mathrm{~g}$ de $\mathrm{MS} /$ min en moyenne). 
TABLEAU 1

Influence du rythme de distribution du foin sur les paramètres du comportement alimentaire.

P4

Par jour :

Quantités de MS ingérées $(\mathrm{g})$

Durée de l'ingestion ( $\mathrm{min}$ )

Vitesse d'ingestion ( $\mathrm{g} \mathrm{MS} / \mathrm{min}$ )

Nombre de repas

Durée de rumination ( $\mathrm{min}$ )

Nombre de cycles de rumina-

tion

Vitesse de mastication ( $\mathrm{g}$

$\mathrm{MS} / \mathrm{min}$ )

Pour le grand repas du matin :

Quantités de MS ingérées ( $\mathrm{g}$ )

Durée d'ingestion ( $\mathrm{min}$ )

Vitesse d'ingestion ( $\mathrm{g} \mathrm{MS} / \mathrm{min}$ )

Poids vif des moutons $(\mathrm{kg})$

$\begin{array}{rrrr}1320 & 1294 & 1358 & \pm 81 \\ 350 & 366 & 320 & \pm 44 \\ 3.76^{\mathrm{a}} & 3.55^{\mathrm{a}} & 4.30^{\mathrm{b}} & \pm 0.51 \\ 6.5^{\mathrm{a}} & 9.0^{\mathrm{b}} & 6.0^{\mathrm{a}} & \pm 1.3 \\ 513 & 528 & 537 & \pm 42 \\ 576 & 574 & 573 & \pm 47 \\ 1.54 & 1.44 & 1.58 & \pm 0.14\end{array}$

$\begin{array}{rrrr}580^{\mathrm{a}} & 759^{\mathrm{b}} & 652^{\mathrm{b}} & \pm 73 \\ 123^{\mathrm{a}} & 180^{\mathrm{b}} & 124^{\mathrm{a}} & \pm 36 \\ 4.86 & 4.65 & 5.29 & \pm 1.08 \\ 75 & 74 & 68 & \end{array}$

N.B. : Sur une même ligne seules les valeurs suivies de lettres distinctes sont significativement différentes $(P<0,05)$.

TABLEAU 2

Répartition journalière des durées d'ingestion et de rumination.

\begin{tabular}{ccccccc}
\hline & \multicolumn{3}{c}{ Durées d'ingestion (min) } & \multicolumn{2}{c}{ Durées de rumination (min) } \\
\cline { 2 - 7 } & $9-17 \mathrm{~h}$ & $17-21 \mathrm{~h}$ & $21-9 \mathrm{~h}$ & $9-17 \mathrm{~h}$ & $17-21 \mathrm{~h}$ & $21-9 \mathrm{~h}$ \\
\hline $\mathrm{P} 2$ & $273^{\mathrm{b}}$ & $46^{\mathrm{b}}$ & $46^{\mathrm{a}}$ & 128 & 93 & 307 \\
$\mathrm{P} 3$ & $147^{\mathrm{a}}$ & $12^{\mathrm{b}}$ & $160^{\mathrm{b}}$ & 140 & 88 & 307 \\
P4 & $163^{\mathrm{a}}$ & $140^{\mathrm{a}}$ & $47^{\mathrm{a}}$ & 129 & 75 & 309 \\
$\begin{array}{c}\text { Ecart-type } \\
\text { résiduel }\end{array}$ & \pm 25 & \pm 29 & \pm 41 & \pm 21 & \pm 31 & \pm 47 \\
\hline
\end{tabular}

N.B. : Sur une même colonne les chiffres suivis de lettres distinctes sont statistiquement différents.

3. Etat de réplétion du réticulo-rumen (tabl. 3, fig. 1 et 2 ).

Pour l'ensemble des 3 périodes étudiées le contenu frais du rumen a été minimum avant le repas du matin. II a augmenté au cours des grands repas pour être maximum après le repas du soir en P1 et P3. En P2 il n'y a pas eu de vidages en fin d'après-midi et il est possible que, malgré une ingestion très élevée lors du grand repas ( $54 \%$ de la journée), les petits repas de l'après-midi aient maintenu assez longtemps entre 11 et $12 \mathrm{~kg}$ le contenu de matière fraîche du rumen. 
Les contenus maxima de matière fraîche ont été égaux respectivement à 18 , 17 et $18 \%$ du poids vif des animaux. La signification des différences observées est donnée dans le tableau 3.

\section{TABLEAU 3}

Influence du rythme de distribution du foin sur les quantités ingérées et l'état de réplétion du réticulo-rumen.

\begin{tabular}{|c|c|c|c|c|c|c|}
\hline & & P1 & $\mathrm{P} 2$ & P3 & $\begin{array}{l}\text { Ecart-type } \\
\text { résiduel }\end{array}$ & $\begin{array}{l}\text { Résultat de } \\
\text { l'analyse } \\
\text { de variance }\end{array}$ \\
\hline \multicolumn{7}{|c|}{ Quantités ingérées $M S$ ( $\mathrm{g} /$ jour) : } \\
\hline \multirow{2}{*}{\multicolumn{2}{|c|}{$\begin{array}{l}\text { totales } \\
\text { lors du grand repas avant } \\
\text { les vidages suivant ce repas }\end{array}$}} & 1313 & 1259 & 1359 & 84 & N.S. \\
\hline & & 481 & 678 & 529 & - & N.S. \\
\hline \multicolumn{7}{|c|}{ Quantités présentes dans le réticulo-rumen (g) } \\
\hline Matière fraîche & $\begin{array}{l}-9 \mathrm{~h} \\
-11 \text { ou } 11 \mathrm{~h} 30 \\
-17 \text { ou } 21 \mathrm{~h} \\
-19 \text { ou } 23 \mathrm{~h}\end{array}$ & $\begin{array}{r}9230^{\mathrm{a}} \\
11335^{\mathrm{b}} \\
11341^{\mathrm{b}} \\
11862^{\mathrm{b}}\end{array}$ & $\begin{aligned} 9329^{\mathrm{a}} \\
11784^{\mathrm{b}} \\
-\end{aligned}$ & $\left.\begin{array}{l}10148^{\mathrm{a}} \\
11146^{\mathrm{ab}} \\
11420^{\mathrm{ab}} \\
12776^{\mathrm{b}}\end{array}\right)$ & 635 & $\begin{array}{r}\text { N.S. } \\
\text { N.S. } \\
\text { N.S. } \\
(\mathrm{P} 1 \neq \mathrm{P} 3)\end{array}$ \\
\hline Matière sèche & $\begin{array}{l}-9 \mathrm{~h} \\
-11 \text { ou } 11 \mathrm{~h} 30 \\
-17 \text { ou } 21 \mathrm{~h} \\
-19 \text { ou } 23 \mathrm{~h}\end{array}$ & $\begin{array}{l}1119^{\mathrm{a}} \\
1543^{\mathrm{b}} \\
1431^{\mathrm{tat}} \\
1603^{\mathrm{b}}\end{array}$ & $\begin{array}{r}870^{\mathrm{a}} \\
1487^{\mathrm{b}} \\
-\quad\end{array}$ & $\left.\begin{array}{l}1133^{\mathrm{a}} \\
1608^{\mathrm{ab}} \\
1469^{\mathrm{ab}} \\
1866^{\mathrm{b}}\end{array}\right\}$ & 172 & $\begin{array}{r}\text { N.S. } \\
\text { N.S. } \\
\text { N.S. } \\
(P 1 \neq P 3)\end{array}$ \\
\hline NDF & $\begin{array}{l}-9 \mathrm{~h} \\
-11 \mathrm{~h} \text { ou } 11 \mathrm{~h} 30 \\
-17 \text { ou } 21 \mathrm{~h} \\
-19 \text { ou } 23 \mathrm{~h}\end{array}$ & $\begin{array}{l}796^{\mathrm{a}} \\
1131^{\mathrm{b}} \\
1060^{\mathrm{ab}} \\
1173^{\mathrm{b}}\end{array}$ & $\begin{array}{r}621^{\mathrm{a}} \\
1069^{\mathrm{b}} \\
-\quad\end{array}$ & $\left.\begin{array}{rl} & 808^{\mathrm{a}} \\
1 & 187^{\mathrm{b}} \\
1 & 051^{\mathrm{ab}} \\
1 & 359^{\mathrm{b}}\end{array}\right)$ & 131 & $\begin{array}{r}\text { N.S. } \\
\text { N.S. } \\
\text { N.S. } \\
(\mathrm{P} 1 \neq \mathrm{P} 3)\end{array}$ \\
\hline \multicolumn{2}{|c|}{ Poids vif des moutons fistulés $(\mathrm{kg})$} & 66 & 70 & 70 & & \\
\hline
\end{tabular}

N.B. : Sur une même colonne, séparément pour la matière fraîche, la matière sèche et le NDF, seules les valeurs suivies de lettres différentes sont significativement différentes.

Les variations des teneurs en matière sèche ont amplifié les écarts relatifs en quantités de matière sèche présentes dans le rumen. En effet ces teneurs sont respectivement égales à $12,1,9,3$ et $11,2 \%$ avant le repas du matin; elles augmentent au cours des repas pour atteindre $12,6 \%$ après l'unique repas en P2 et, respectivement 13,6 et $14,6 \%$ après les repas du soir en P1 et P3. Dans ces conditions les quantités de matière sèche présentes dans le réticulo-rumen ont augmenté fortement au cours de la journée $(+484 \mathrm{~g}$ en $\mathrm{P} 1 ;+617 \mathrm{~g}$ en P2 et $+733 \mathrm{~g}$ en $\mathrm{P} 3$ ), mais ont diminué légèrement entre les deux distributions en $\mathrm{P} 1$ et $\mathrm{P} 3$ alors que les quantités de matière fraîche restaient stables. Les quantités de 


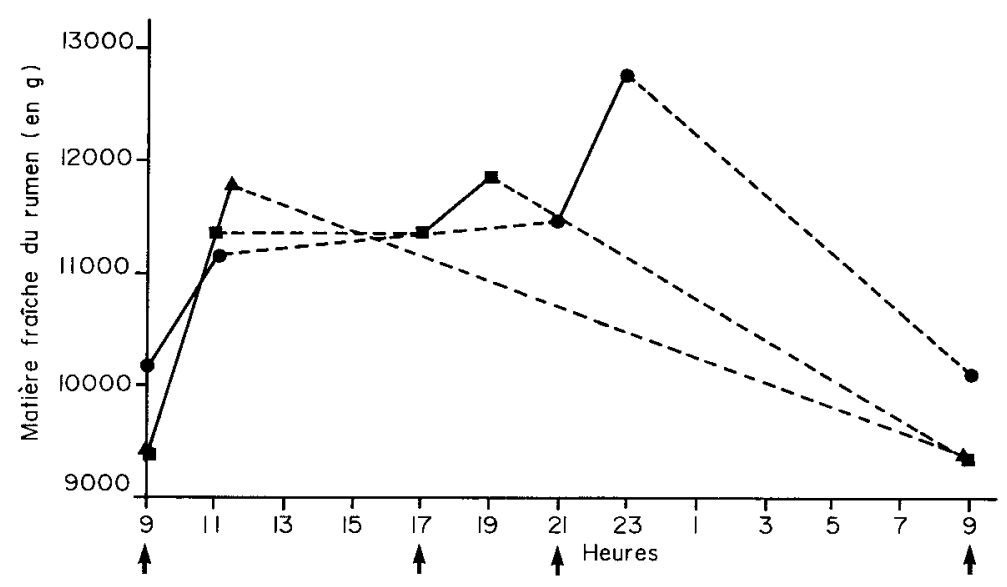

FIG. 1. Evolution, au cours du nycthémère, de la quantité de matière fraîche du rumen.
- période 3 .

Les flèches indiquent les heures de distribution des repas.

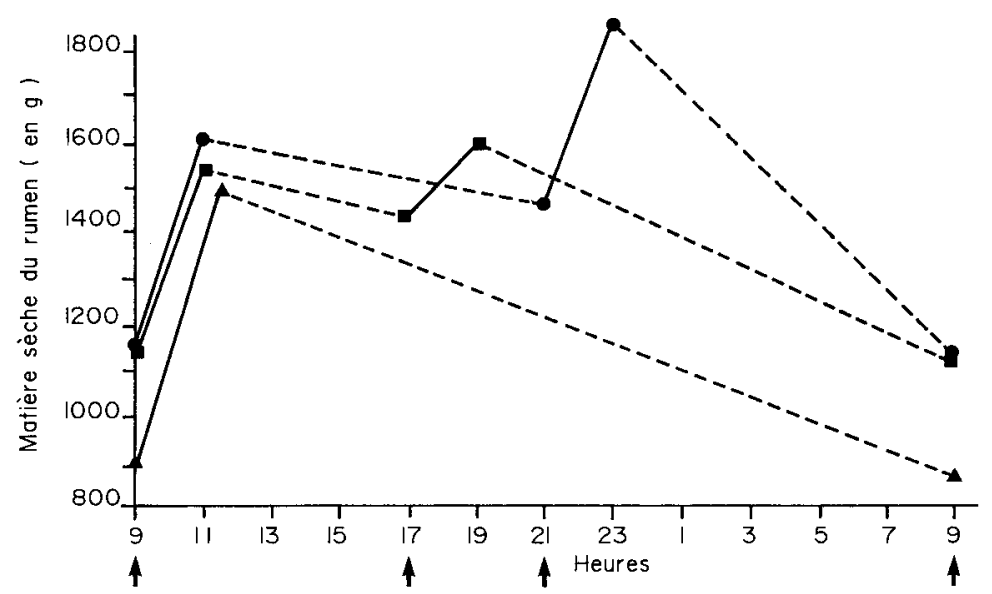

FIG. 2. - Evolution de la quantité de matière sèche au cours du nycthémère.

période $1 ; \Delta-\Delta$ période $2 ; 0$ période 3.

parois (NDF) ont suivi une évolution parallèle car les teneurs correspondantes ont peu varié au cours de la journée : $71,3 \%$ de la MS en moyenne à $9 \mathrm{~h}$ et $72,6 \%$ en moyenne lors du dernier vidage.

Compte tenu des résultats observés il n'y a donc pas d'influence nette du rythme de distribution. On peut simplement noter que les quantités de contenu restent élevées et comparables aux différentes heures de vidage en P1 et P3. La quantité de contenu après le repas du soir est plus élevée en P3 qu'en P1. 
Enfin il est possible d'estimer, en moyenne, à titre indicatif, les vitesses de disparition des digesta hors du rumen (Baumont, Dulphy et Andrieu, 1988). On observe alors :

- une vidange lente au cours du repas du matin (environ $35 \mathrm{~g}$ de $\mathrm{MS} / \mathrm{h}$ ) ;

- une vidange rapide lors du repas du soir (146 g de MS/h en P1, mais 52 en P3) :

- des vidanges sur la journée comparables d'un traitement à l'autre (respectivement 55,52 et $57 \mathrm{~g}$ de $\mathrm{MS} / \mathrm{h}$ en P1, P2 et P3).

\section{Discussion.}

Dans notre essai le rythme de distribution du fourrage n'a donc pas influencé significativement les quantités de foin ingérées par jour. Le fait de distribuer le repas du soir 8 ou $12 \mathrm{~h}$ après le repas du matin ne change pas le niveau d'ingestion. Le fait de donner le foin en une seule fois a cependant tendance à faire baisser les quantités ingérées. Cette observation devra être vérifiée car elle peut être liée à la nature du régime. Elle pourrait être différente avec un régime très ingestible.

L'augmentation de la quantité ingérée après l'unique distribution et celle des petits repas chez les moutons ne recevant le foin qu'une fois, indiquent que les moutons peuvent modifier la répartition de leurs activités pour ingérer des quantités proches de celles ingérées normalement. Le fait de distribuer la nourriture est un élément structurant important pour les activités alimentaires de l'animal grâce à la stimulation qu'il exerce sur celui-ci (Gatel, 1984). A la suite d'une distribution l'animal fait un grand repas. La multiplication des distributions réduit donc le nombre de petits repas. A l'inverse, l'absence d'une seconde distribution n'empêche pas l'animal de manger l'après-midi, ce qui rejoint les observations de Metz (1975) et de Nocek et Braund (1985). Dans notre essai, les quantités ingérées l'après-midi ne sont cependant pas très élevées, à cause peut-être de la faible vitesse de digestion du foin, et donc de sa faible vitesse de disparition hors du rumen.

L'évolution au cours de la journée des quantités de contenu dans le réticulo-rumen est conforme à celle trouvée par différents auteurs (Balch, 1958 ; Dulphy, Béchet et Thomson, 1975 ; Thomson et al., 1985 ; Baumont, Dulphy et Andrieu, 1988). Avec deux grands repas par jour le rumen est plus rempli à la fin du repas du soir qu'à celle du repas du matin. Lors d'une seule distribution de foin, il y a peu de digesta dans le rumen avant l'affouragement et le contenu est très liquide. Associé au fait que les animaux ont probablement plus faim, on comprend qu'ils ingèrent alors plus pendant le grand repas du matin. La quantité de matière sèche quittant pendant ce temps le rumen est faible et le niveau de contenu frais en fin de repas atteint celui observé en fin de repas le soir, valeur pouvant être considérée comme maximale dans nos conditions d'alimentation. L'absence d'un grand repas le soir doit expliquer la faible quantité de digesta présente le matin, les petits repas n'encombrant pas le rumen comme les grands.

Lorsque les deux distributions de foin ont été espacées de $12 \mathrm{~h}$ au lieu de $8 \mathrm{~h}$, la quantité de contenu a été identique dans la journée, mais plus élevée après le repas du soir. Dans ces conditions, même si des écarts ne sont pas significatifs, 
avec le rythme de distribution $2 \times 12 \mathrm{~h}$ le rumen a été plus plein après les grands repas le soir que le matin. Cela est en opposition avec les observations de Balch, Reid et Stroud (1957) sur bovins et est probablement dû au fait que nos moutons ont ruminé moins pendant la journée que la nuit, la rumination accélérant la vidange du rumen (Dardillat et Ferlay, non publié). Il est vrai que les animaux n'ont pas été éclairés en permanence et ont sans doute conservé une répartition normale de leur rumination. Notre deuxième hypothèse n'est donc pas vérifiée et nous ne pouvons pas, dans les conditions de cet essai, lors d'un affouragement toutes les 12 heures, extrapoler à la nuit les résultats trouvés le jour.

Pour ce qui concerne la régulation des quantités ingérées, nous vérifions donc que le niveau de remplissage du rumen est variable au cours du nycthémère et que les animaux peuvent être rassasiés alors que l'encombrement de leur réticulo-rumen n'est pas au maximum. Avec un affouragement par jour ce maximum est évidemment atteint rapidement, sinon il l'est après le repas du soir. Dans notre essai, avec deux grands repas par jour, les quantités maximales observées de matière fraîche présentes dans le rumen ont été très proches selon les deux modalités de distribution, si on corrige pour le poids des animaux, soit 177 et $182 \mathrm{~g} / \mathrm{kg}$ de poids vif contre seulement $168 \mathrm{~g}$ avec une seule distribution. Retenons comme valeur maximale observée $180 \mathrm{~g} / \mathrm{kg}$. II sera intéressant de confirmer par d'autres essais si la valeur plus faible obtenue en $\mathrm{P} 2$ est significative alors qu'elle ne l'est pas ici. Mais il est intéressant aussi de la comparer avec celle obtenue pour d'autres foins. Dulphy, Béchet et Thomson (1985) trouvent $157 \mathrm{~g} / \mathrm{kg}$ de poids vif pour un foin ventilé de brome ayant une digestibilité élevée (DMO $=0,71)$, mais une ingestibilité faible $\left(48 \mathrm{~g}\right.$ de $\mathrm{MS} / \mathrm{kg} \mathrm{P}^{0,75}$ ) avec seulement 15,5 g au plus de MS de contenu par $\mathrm{kg}$ de poids vif contre 25,5 g dans cet essai. Baumont, Dulphy et Andrieu (1988) trouvent une quantité maximale observée de $175 \mathrm{~g}$ de matière fraîche et $21 \mathrm{~g}$ de MS par $\mathrm{kg}$ de poids vif pour un foin de graminées ayant une ingestibilité de $47 \mathrm{~g}$ de $M S / \mathrm{kg}^{0,75}$ et une digestibilité de 0,59.

Nous observons donc d'un essai à l'autre de fortes variations du poids du contenu de rumen, mais aussi, à même niveau de poids frais, des différences de poids sec. Cela rejoint les résultats de nombreuses études faites sur le contenu réticulo-omasal. En effet depuis la revue de Balch et Campling (1962), de nombreuses études ont envisagé le rôle de l'état de réplétion du réticulo-rumen sur les quantités ingérées. Citons parmi d'autres celles de Montgomery et Baumgardt (1965), Grovum (1979), Forbes (1980), Hidari (1984). Dans un premier temps on s'est efforcé de démontrer le rôle fondamental de l'encombrement du rumen, soit en augmentant cet encombrement, soit en le diminuant artificiellement (Campling et Balch, 1961; Grovum, 1979; Hidari, 1984). Simultanément, on a mesuré le niveau de remplissage du rumen, en général à un moment fixe de la journée (Jarrige, 1978). Dans un deuxième temps, les auteurs se sont intéressés à l'évolution journalière du contenu ruminal (Tulloh, Hugues et Newth, 1965 ; Dulphy, Béchet et Thomson, 1975 ; Paquay, de Baere et Lousse, 1971 ; Hidari, 1981 ; Thomson et al., 1985). II ressort de toutes ces études que le rôle de l'état de réplétion du rumen est important pour la régulation de l'appétit, mais que les phénomènes explicatifs sont plus complexes que ne le pensaient les 
premiers auteurs. En effet, le niveau moyen de remplissage du réticulo-rumen varie beaucoup selon les aliments et les situations et le niveau maximal observé, variable aussi, n'est atteint probablement que pendant de courtes périodes. II existe donc au cours du nycthémère des phénomènes de rassasiement d'ordre physiologique par le fait que le rumen n'est pas plein en permanence, d'où le modèle de Forbes (1980) pour prévoir les activités alimentaires et les quantités journalières ingérées. II n'en demeure pas moins que pour améliorer de tels modèles de prévision il faut connaître les facteurs de variation du contenu récitulo-omasal. En outre, la mesure de ce contenu permet d'évaluer la vitesse de transit des digesta hors du rumen qui détermine aussi directement le niveau d'ingestion.

En définitive, le rythme d'affouragement joue un rôle très important sur l'organisation de l'ingestion dans la journée, mais avec un foin peu ingestible on peut distribuer le fourrage une seule fois par jour sans modifier notablement son niveau d'ingestion. Dans ce cas on observe alors un grand repas très long puis un nombre élevé de petits repas. Ce système doit permettre de suivre avec peu de vidages l'évolution du contenu réticulo-omasal, à condition de vérifier que le maximum observé se situe bien après l'unique grand repas. Avec deux distributions par jour, le fait d'espacer les grands repas de $12 \mathrm{~h}$ n'autorise pas la limitation des observations aux heures de jour, mais là aussi il reste à vérifier si un éclairage continu et un meilleur isolement des animaux ne le permettrait pas.

Reçu en décembre 1987. Accepté en avril 1988.

\section{Références}

BALCH C. C., 1958. Observations on the act of eating in cattle : Brit. J. Nutr., 12, 330-345.

BALCH C. C., CAMPLING R. C., 1962. Regulation of voluntary food intake in ruminants. Nutr. Abstr. Rev., 32, 669-686.

BALCH C. C., REID J. T., STROUD J. W., 1957. Factors influencing the rate of excretion of administered chromium sesquioxide by steers. Brit. J. Nutr., 11, 184-197.

BAUMONT R., DULPHY J. P., ANDRIEU J. P., 1988. Comportement alimentaire et état de réplétion du réticulo-rumen chez le mouton nourri à volonté de foin de prairie ou de luzerne, avec accès continu ou limité : incidences sur le contrôle physique de l'ingestion. Reprod. Nutr. Dévelop. 28, 573-588.

CAMPLING R. C., BALCH C. C., 1961. Factors affecting the voluntary intake of food by cows. 1 Preliminary observations on the effect, on the voluntary intake of hay, of changes in the amount of the reticulo-rumen contents. Brit. J. Nutr., 15, 523-530.

CHASE L. E., WANGNESS P. J., BAUMGARDT B. R., 1976. Feeding behaviour of steers fed a complete mixed ration. J. Dairy Sci., 59, 1923-1928.

DULPHY J. P., BÉCHET G., THOMSON E., 1975. Influence de la structure physique et de la qualité de conservation des ensilages de graminées sur leur ingestibilité. Ann. Zootech., 24, 81 -94.

DULPHY J. P., REMOND B., THERIEZ M., 1979. Ingested behaviour and related activities in ruminants, 103-122. In RUCKEBUSCHY. and THIVEND P. Digestion and metabolism in ruminants, MTP Press, Lancaster, England.

FORBES J.M., 1980. A model of the short-term control of feeding in the ruminant: effects of changing animal and feed characteristics. Appetite, 1, 21-41

GATEL F., 1984. Signification de la satiété à court terme chez le mouton : influence de la qualité du fourrage et des stimuli associés à la prise de nourriture. Ann. Zootech., 33, 111-118.

GOERING H. K., VAN SOEST P. J., 1970. Forage fiber analyses. Agriculture Handbook $\mathrm{n}^{\circ} 379$. Agric. Res. Serv., US Dept Agric., 20 p. 
GROVUM W. L., 1979. Factors affecting the voluntary intake of food by sheep. 2 - The role of distension and tactile input from the compartments of the stomach. Brit. J. Nutr., 42, 425-436.

HIDARI H., 1981. The relationships between rumen load and diurnal pattern of sheep fed in various time of acces to feed. Jap. J. Zoot. Sci., 52, 219-226.

HIDARI H., 1984. Effect of altering the rumen load on the feeding behavior in sheep. Can. J. anim. Sci., 64 (suppl.), 320-321.

JARRIGE R., 1978. Digestion. In Alimentation des ruminants. Ed. I.N.R.A. Publ., Route de Saint-Cyr 78000 Versailles.

METZ J. H. M., 1975. Time patterns of feeding and rumination in domestic cattle. Meded. Landbouwhogesch. Wageningen, 75-12.

MICHALET-DOREAU B., GATEL F., 1984. Evolution au cours d'une année des quantités de foin ingérées par des béliers castrés. Ann. Zootech., 32, 459-464.

MONTGOMERY M. J., BAUMGARDT B. R., 1965. Regulation of food intake in ruminants. 1 Pelleted rations varying in energy concentration. J. Dairy Sci., 48, 569-574.

NOCEK J. E., BRAUND D. G., 1985. Effect of feeding frequency on diurnal dry matter and water consumption, liquid dilution rate, and milk yield in first lactation. J. Dairy Sci., 68, 2238-2247.

PAQUAY R., de BAERE R., LOUSSE A., 1971. Des variations du poids vif chez la bête bovine. Rev. Agric., 4, 419-429.

THOMSON R. F., CRUICKSHANG G. J., POPPI D. P., SYKES A. R., 1985. Diurnal patterns of rumen fill in grazing sheep. Proc. N. Z. anim. Prod., 45, 117-120.

TULLOH N. M., HUGHES J. W., NEWTH R. P., 1971. Physical studies of the alimentary tract of grazing cattle. I-Measurement of volume of water in the reticulo-rumen. N. Z. J. agric. Res., 8, 636-651.

ULYATT M. J., WAGHORN G. C., JOHN A., REID C. S. W., MONRO J., 1984. Effect of intake and feeding frequency on feeding behaviour and quantitative aspects of digestion in sheep fed chaffed lucerne hay. J. agric. Sci. Camb., 102, 645-657. 